\title{
Formación del profesorado a distancia sobre aprendizaje- servicio: aspectos a considerar
}

\author{
Beatriz Ortega-Ruipérez \\ Universidad Internacional de La Rioja, España
}

\section{Resumen}

La formación de docentes debe ser práctica y aplicada, de modo que aprendan cómo implementar diferentes técnicas educativas en sus clases, como ocurre con el aprendizaje-servicio. Si, además, la formación es online, pueden surgir inconvenientes para su correcta difusión, como que los docentes no valoren el aprendizaje-servicio o no sepan cómo aplicarla. Por esta razón, resulta importante estudiar y planificar las experiencias formativas en línea para docentes. Por tanto, el objetivo de este estudio es mejorar la difusión del aprendizaje-servicio entre los futuros docentes para conseguir que se aplique en más aulas. Para ello, se va a evaluar la opinión de profesores en formación, tras impartir una breve experiencia que permita conocer los aspectos más prioritarios en una formación de este tipo. Se va a utilizar un cuestionario para valorar el conocimiento sobre aprendizaje-servicio y la percepción de estos docentes sobre los beneficios e inconvenientes de esta práctica educativa. Los resultados muestran que los docentes valoran la parte práctica de la formación como lo más positivo para comprender los beneficios de esta técnica, pero valoran por debajo el desarrollo de algunas habilidades y los materiales disponibles para aplicar el aprendizaje-servicio. Estos resultados sirven para reflexionar sobre los aspectos en los que debe centrarse en el futuro una formación online sobre aprendizaje-servicio.

\section{Palabras clave}

Aprendizaje social, enseñanza a distancia, método de proyectos, formación de profesores, aprendizaje-servicio.

Fecha de recepción: 30/VII/2019

Fecha de aceptación: 28/VIII/2019 


\title{
Online teacher training in service-learning: aspects to consider
}

\begin{abstract}
Teacher training should be practical and applied, so that teachers learn how they should apply different educational techniques in their classes, as is the case with service-learning. If, however, training takes place online, some difficulties may arise in the promotion of service-learning, such as, for example, teachers not valuing servicelearning enough or not knowing how to implement it in their teaching. For this reason, it is important to study and properly plan online training experiences for teachers. Therefore, the objective of this study is to improve the promotion of service-learning among future teachers in order to extend its use to more classrooms. For this purpose, the opinion of teachers in training was evaluated, after they took part in a brief experience that showed them the most important aspects in a training of this type, with a view to its subsequent improvement. The results show that teachers value the practical part of the training as the most positive aspect for understanding the benefits of this technique; however, they give a low rating to the development of certain skills and the materials available to apply service-learning. These results will help us reflect on the aspects that an online training in service-learning should focus on in the future.
\end{abstract}

\section{Keywords}

Social learning, distance study, project method, teacher education, service-learning. 


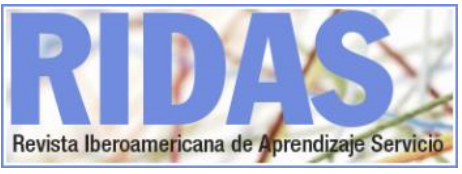

grado de educación primaria.

\section{Introducción}

En los últimos años están proliferando las iniciativas y proyectos sobre aprendizaje-servicio. Este enfoque educativo, que vincula servicios sociales con aprendizaje, permite que los alumnos realicen proyectos reales con impacto social (Martín et al., 2018). Como señalan Puig, Gijón, Martín y Rubio (2011) esto permite una educación más inclusiva y sirve para un aprendizaje por competencias, ya que se realiza un aprendizaje reflexivo de conocimientos, habilidades y valores.

Desde la universidad debemos promover proyectos que vayan más allá del aprendizaje, debemos formar a una sociedad concienciada para la justicia social, por lo que resulta necesario utilizar enfoques educativos como el aprendizaje-servicio. Si, además, las personas a las que formamos van a encargarse de la enseñanza de las generaciones futuras, debemos plantearnos dos objetivos. Por una parte, que entiendan por qué este enfoque educativo debe ser un eje central en el proyecto educativo de cualquier centro $y$, por otra parte, que conozcan cómo aplicar el aprendizajeservicio en su aula de manera efectiva con sus alumnos.

\section{Existen bastantes propuestas para} incluir el aprendizaje-servicio en la formación de docentes, como la de Fuertes (2013) en el prácticum del profesorado, la de Rodríguez (2014) sobre la importancia de incluirla en el grado de pedagogía, o las de Martínez (2018), y López y Benítez (2018) en el
Sin embargo, la formación online sobre aprendizaje-servicio conlleva unas pautas propias, por lo que, si la instrucción se realiza online, se deben tener en cuenta una serie de aspectos que garanticen que la formación cumple con los dos objetivos antes

mencionados. Los docentes en este caso únicamente reciben una formación a distancia. Sin embargo, tendrán que ponerla en práctica de forma presencial con sus alumnos.

Con este estudio se va a valorar la calidad percibida de una formación online sobre aprendizaje-servicio, por parte de los docentes en formación en línea, que permita reducir las limitaciones respecto a cualquier formación presencial, y que cualquier formador de formadores de educación superior a distancia pueda aplicar en su aula virtual. En concreto, esta experiencia se ha diseñado para llevar a cabo dos sesiones sobre el aprendizajeservicio, partiendo de la limitación de que este contenido suele ser una pequeña parte de los contenidos de una asignatura.

La primera sesión, de explicación sobre esta práctica educativa, pretende una difusión adecuada de la misma, haciendo hincapié sobre los beneficios que puede aportar y sobre cómo se pueden superar algunos inconvenientes que puedan surgir al aplicarla. La segunda sesión consiste en una sesión práctica, de simulación de un proyecto de aprendizaje-servicio, en la que experimentan las diferentes fases a partir de analizar su propio entorno, el de los docentes en formación, durante 
una semana.

Además, como destaca Puig (2018), para una correcta difusión del aprendizaje-servicio es importante, entre otras cuestiones, poder crear las condiciones necesarias para que las entidades sociales ofrezcan espacios de servicio adecuados, y para promover sinergias entre la sociedad y las administraciones. Sin embargo, los alumnos de la formación en línea pueden residir en diferentes partes del mundo, con lo que sería imposible crear una red en un entorno social concreto.

En este caso, se nos presenta un desafío, que es el de crear conciencia sin poder aplicar un proyecto real de aprendizaje-servicio, por lo que se apuesta por mostrar la iniciativa de Design for Change a través de las páginas web de cada país, en las que se pueden visualizar proyectos ya realizados y aparece el contacto de los coordinadores de cada región.

\section{Marco teórico}

El aprendizaje-servicio es una práctica educativa, basada en proyectos, que permite aplicar los contenidos curriculares en situaciones reales de compromiso social. Como afirman Pérez y Ochoa (2017) a los alumnos que llevan a cabo este tipo de práctica educativa les permite "ser protagonistas activos al implicarse en las necesidades de su entorno con la finalidad de mejorarlo" (p.175).

Esto implica que son los propios alumnos quienes deben decidir cuál va a ser el propósito de su proyecto de aprendizaje-servicio, ya que la implicación en un proyecto impuesto será menor que en un proyecto elegido. Por tanto, aspectos como la elección del proyecto por parte de los alumnos, son fundamentales para que la aplicación de esta técnica sea adecuada.

2.1 Design for Change: la iniciativa de aprendizaje-servicio a nivel mundial

Para facilitar la aplicación del aprendizaje-servicio existe una iniciativa internacional que establece diferentes fases en el proyecto. Estas fases se pueden utilizar en la propia formación a distancia, y aunque no todas se podrán poner en práctica debido a las características de esta formación, se debe buscar un equilibrio entre la práctica real y la simulación.

Design for Change es una iniciativa de aprendizaje-servicio a nivel mundial que se inició en India, en 2009.

Actualmente, 10 años después, cuenta con la participación de más de 65 países y regiones (DFC World, s.f.). Esta iniciativa ha creado un marco de aplicación que cuenta principalmente con 4 fases: siente, imagina, haz y comparte. Esta iniciativa se propone a través de estas cuatro fases para facilitar la aplicación del aprendizajeservicio en cualquier aula de cualquier parte del mundo.

Cada una de las fases propuestas en Design for Change cuenta con unas acciones determinadas que permiten a los alumnos avanzar a través del problema hasta la realización de un proyecto. Para que los profesores puedan llevar a cabo esta experiencia sin problemas, la web dispone unas guías y vídeos para docentes que 
faciliten el trabajo y desarrollo de habilidades través de este tipo de proyectos (Fisch, McLeod y Bronman, 2013).

\subsection{La formación a distancia}

La formación del aprendizaje-servicio en línea es conocida como eAprendizaje-servicio. El eAprendizajeservicio corresponde a una experiencia de aprendizaje-servicio con algún componente online, pudiendo ser la instrucción o el servicio (Waldner, McGorry y Widener, 2012), en este caso la instrucción.

La mayoría de literatura sobre eAprendizaje-servicio no trabaja la enseñanza online del aprendizajeservicio como técnica educativa, es decir, no hay estudios sobre cómo enseñar en línea a futuros docentes sobre cómo aplicar el aprendizajeservicio con sus alumnos. Las propuestas que se recogen en la investigación aplican esta técnica a disciplinas diferentes, como Bellas Artes, Biblioteconomía, Biología, Derecho, Economía o Enfermería (Esparza, Morín y Rubio, 2018).

Las investigaciones revisadas no coinciden sobre si el aprendizajeservicio online ofrece la experiencia necesaria para el aprendizaje de aprendizaje-servicio o, por el contrario, los alumnos a distancia pueden obtener concepciones erróneas a través de las experiencias online (McWhorter, Delello y Roberts, 2016).

Por una parte, existen resultados a favor de la factibilidad de las experiencias $100 \%$ online, como los de
Waldner, McGorry y Widener, (2010). Por otra parte, estudios como los realizados por Malvey, Hamby y Fottler (2006) sugieren que la pérdida de experiencias in situ afecta, o el realizado por Lehman y Conceição (2010) sobre la falta de autorreflexión en situaciones de aprendizaje online, clave en el aprendizaje-servicio (Páez y Puig, 2013).

La falta de acuerdo perjudica el rápido desarrollo de una formación online de calidad sobre el aprendizaje-servicio, aunque en este estudio se va a optar por una visión positiva sobre la factibilidad de las experiencias online, pero teniendo en cuenta que es muy fácil caer en la pérdida de la efectividad si no se realiza correctamente. Para ello, se lleva a cabo un apoyo continuo que guía la experiencia a los alumnos, ayudándoles a reflexionar sobre qué conlleva esta práctica educativa en cada una de las fases propuestas en Design for Change.

2.3 Valoración docente sobre el empleo del aprendizaje-servicio

El enfoque del aprendizaje-servicio resulta idóneo para desarrollar habilidades de trabajo en equipo y competencias cívicas, como recogen entre otros muchos Hébert y Hauf (2015) o Miyazaki, Anderson y Jones (2017). Los docentes en formación tienen que conocer las posibilidades de esta técnica y ser capaces de aprovecharla para el desarrollo de habilidades en sus alumnos.

Tras realizar un proyecto de aprendizaje-servicio, la valoración que hacen los propios estudiantes sobre la

Ortega-Ruipérez, B. (2020). Formación del profesorado a distancia sobre aprendizaje-servicio: aspectos a considerar. RIDAS, Revista Iberoamericana de Aprendizaje Servicio, 9, 102-122. 
formación personal y sobre el desarrollo de habilidades profesionales suele ser alto, aunque la valoración puede variar dependiendo de las características de cada proyecto: de su diseño, planificación y ejecución (Caire, 2019).

Estudios como el de Duque (2018) muestran la mayor sensibilidad social de los alumnos después de realizar prácticas de aprendizaje-servicio. Por su parte, Sotelino, Santos y García (2019) estudian el desarrollo de competencias interculturales, detectando mayores expectativas de los alumnos sobre sus competencias cívicosociales.

En cualquier caso, de acuerdo con Strait, Turk y Nordyke (2015), para su enseñanza se necesita reflexionar sobre cómo va a ser la experiencia, una reflexión que permita definir la situación de partida de los estudiantes respecto a estas habilidades y a través de qué actividades lograrán desarrollar las habilidades a un nivel adecuado para su edad y desarrollo. Sólo de esta forma conseguiremos desarrollar competencias, con esta o con cualquier técnica educativa.

Por otra parte, no se han encontrado valoraciones previas en estudios de formación de docentes sobre dificultades para la aplicación de esta técnica docente. Se va a incluir como dimensión del estudio para abrir una nueva línea de investigación.

\section{Metodología}

\subsection{Diseño de la investigación}

Este estudio pretende ser el inicio de una investigación más amplia sobre cómo enseñar a docentes en activo técnicas instruccionales con impacto social, como es el aprendizaje-servicio, en contextos online donde la aplicación de estos proyectos resulta mucho más complicada. Por tanto, este artículo consiste en un estudio exploratorio que nos permitirá construir una base para mejorar la enseñanza del aprendizajeservicio online, en contextos educativos en los que los alumnos dispongan de sesiones presenciales síncronas, pero no sea obligatorio asistir a dichas sesiones presenciales.

Para construir esta base, se han delimitado unas dimensiones que nos permitirán analizar de forma más objetiva cómo perciben y valoran el aprendizaje-servicio los docentes que reciben una formación online. Estas dimensiones son conocimiento general sobre aprendizaje-servicio, valoración de los beneficios del aprendizajeservicio y valoración de los inconvenientes del aprendizaje-servicio.

El estudio se basa en una experiencia educativa que comprende dos sesiones síncronas en las que cada alumno se conecta a la clase desde su domicilio. La primera sesión se dedica a explicar las características del aprendizajeservicio, insistiendo en los beneficios que tiene para los alumnos cada una de las fases, y en cómo se pueden superar algunas dificultades que puede conllevar esta técnica educativa. La segunda sesión es una sesión práctica para que ellos mismos lleven a cabo la realización de un proyecto aprendizajeservicio en clase.

Se adopta un enfoque cuantitativo

Ortega-Ruipérez, B. (2020). Formación del profesorado a distancia sobre aprendizaje-servicio: aspectos a considerar. RIDAS, Revista Iberoamericana de Aprendizaje Servicio, 9, 102-122. 
basado en estadística descriptiva, que pretende servir para generar una idea general sobre la percepción que tienen los docentes en formación online sobre el aprendizaje-servicio, de manera que nos permita mejorar la formación de futuros docentes.

\subsection{Participantes}

Los participantes de esta investigación son cinco grupos del Máster de Tecnología Educativa y Competencias Digitales, de la Universidad Internacional de La Rioja con sede principal en España y varias sedes en diferentes países de Latinoamérica. En total, en estos cinco grupos de alumnos hay matriculados 185 alumnos. Todos los alumnos que acceden al máster deben tener un título en educación superior (grado, diplomatura, licenciatura, ingeniería técnica, etc.), siendo la mayoría de ellos docentes en distintas etapas educativas, desde educación infantil hasta educación superior.

La experiencia práctica de aprendizajeservicio se realizó en dos asignaturas diferentes. Dos de los grupos realizaron la experiencia en la asignatura de Programación y Robótica para Docentes, siendo un total de 97 alumnos matriculados. Los alumnos de la asignatura de Programación y Robótica están en su totalidad matriculados en España. Los tres grupos restantes realizaron la experiencia de aprendizaje-servicio en la asignatura de Emprendimiento, Innovación y Creatividad Digital, siendo un total de 88 alumnos matriculados en la asignatura. La totalidad de estos alumnos están matriculados en

\section{Ecuador.}

La respuesta al cuestionario fue voluntaria para todos aquellos alumnos que quisieran participar. La participación se premió con una recompensa en la calificación final de $+0,1$. La participación finalmente alcanzó un $35 \%$ del total de los alumnos matriculados en ambas asignaturas, esto es 65 alumnos.

\subsection{Instrumentos y materiales}

El instrumento que se ha utilizado para recoger la información necesaria para lograr los objetivos es un cuestionario realizado específicamente para el estudio. Se ha elegido la creación de un cuestionario propio con un total de 38 preguntas, dado que los cuestionarios revisados durante la investigación (De Castro, Martínez y Guerra, 2017; Toncar, Reid, Burns, Anderson y Nguyen, 2006) no respondían adecuadamente a todas las dimensiones del estudio.

Para responder al propósito de la investigación se necesitaba un cuestionario sencillo, rápido y fácil de contestar, en el que los alumnos pudieran responder a preguntas que abarcasen toda la investigación, ya que se trata de una investigación exploratoria y se consideró que elegir un cuestionario estandarizado podría desmotivar a los participantes, ya que son más extensos, y esto afectaría a la fiabilidad de sus respuestas.

Para diseñar el cuestionario se han elegido las preguntas en base a los cuestionarios revisados, de forma que se garantice un mínimo de fiabilidad.

Ortega-Ruipérez, B. (2020). Formación del profesorado a distancia sobre aprendizaje-servicio: aspectos a considerar. RIDAS, Revista Iberoamericana de Aprendizaje Servicio, 9, 102-122. 
Las respuestas se recogen con una escala tipo Likert con diferente número de opciones según la sección concreta del cuestionario, debido al carácter multidimensional del cuestionario, que ha sido realizado a partir de diferentes fuentes. El cuestionario tiene en total cinco secciones:

- Sección 1. Aspectos importantes para mi aprendizaje. Preguntas sobre cómo de importante es para el docente unas afirmaciones sobre el aprendizaje significativo y desarrollo de habilidades (tabla 1). Escala de respuesta de 1 a 7 , siendo 1 la respuesta más baja posible y 7 la más alta.

- Sección 2. Mi experiencia con EL aprendizaje-servicio. Preguntas sobre la experiencia de aprendizaje-servicio realizada en clase (tabla 2). Escala de 1 a 5.

- Sección 3. Enseñanza desde la perspectiva de aprendizajeservicio. Preguntas sobre la percepción de utilidad de la enseñanza aprendizaje-servicio (tabla 3). Escala de 1 a 5.

- Sección 4. Situación laboral actual: trabajo en educación / no trabajo en educación. Preguntas sobre las posibilidades reales de aplicación según su situación (tabla 4). Escala de 1 a 5.

- Sección 5. Para terminar. Pregunta final de valoración global de la experiencia y de la técnica aprendizaje-servicio (tabla 5). Escala de 1 a 10.
Estas secciones contienen preguntas de todas las dimensiones que se quieren estudiar y que se pueden ver en la figura 1.

Figura 1. Dimensiones y subdimensiones de estudio

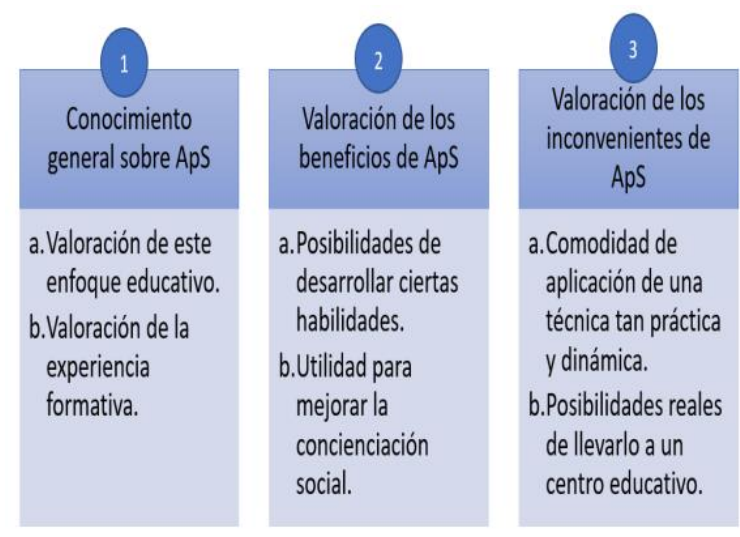

Fuente: elaboración propia

En las tablas $1-5$ se pueden consultar todas las preguntas incluidas en las diferentes secciones del cuestionario y la dimensión de estudio a la que pertenece cada una de las preguntas.

Por último, a modo de resumen, en la tabla 6 se muestran los números de las preguntas incluidas en cada dimensión del estudio.

Tabla 1. Preguntas de la Sección 1 del cuestionario de investigación

\begin{tabular}{ccc}
\hline No & Preguntas & $\begin{array}{c}\text { Dimensión } \\
\text { de estudio }\end{array}$ \\
\hline $\mathbf{1}$ & $\begin{array}{l}\text { Aplicar el conocimiento al } \\
\text { mundo real }\end{array}$ & $1 \mathrm{a}$ \\
$\mathbf{2}$ & $\begin{array}{l}\text { Desarrollar habilidades de } \\
\text { trabajo }\end{array}$ & $2 \mathrm{2a}$ \\
\hline
\end{tabular}

Ortega-Ruipérez, B. (2020). Formación del profesorado a distancia sobre aprendizaje-servicio: aspectos a considerar. RIDAS, Revista Iberoamericana de Aprendizaje Servicio, 9, 102-122. 


\begin{tabular}{|c|c|c|}
\hline 3 & $\begin{array}{l}\text { Desarrollar habilidades de } \\
\text { organización }\end{array}$ & $2 a$ \\
\hline 4 & $\begin{array}{l}\text { Entender las diferencias } \\
\text { culturales y raciales }\end{array}$ & $2 b$ \\
\hline 5 & $\begin{array}{l}\text { Desarrollar habilidades de } \\
\text { responsabilidad social y } \\
\text { ciudadana }\end{array}$ & $2 b$ \\
\hline 6 & $\begin{array}{l}\text { Desarrollar habilidades para } \\
\text { marcar una diferencia en la } \\
\text { comunidad }\end{array}$ & $2 b$ \\
\hline 7 & $\begin{array}{l}\text { Desarrollar una confianza } \\
\text { social }\end{array}$ & $2 b$ \\
\hline 8 & $\begin{array}{l}\text { Desarrollar habilidades para } \\
\text { asumir la responsabilidad } \\
\text { individual }\end{array}$ & $2 b$ \\
\hline 9 & $\begin{array}{l}\text { Desarrollar habilidades para } \\
\text { ganarse la confianza de los } \\
\text { demás }\end{array}$ & $2 b$ \\
\hline 10 & $\begin{array}{l}\text { Desarrollar habilidades de } \\
\text { liderazgo }\end{array}$ & $2 a$ \\
\hline 11 & $\begin{array}{l}\text { Desarrollar habilidades de } \\
\text { comunicación }\end{array}$ & $2 a$ \\
\hline
\end{tabular}

Nota: Sección 1. Aspectos importantes para mi aprendizaje. Indica cómo de importante es para ti lo siguiente según tu experiencia educativa con el Máster, para que tu aprendizaje sea más significativo.

Fuente: elaboración propia

Tabla 2. Preguntas de la Sección 2 del cuestionario de investigación

\begin{tabular}{|c|c|c|}
\hline No & Preguntas & $\begin{array}{l}\text { Dimensión } \\
\text { de estudio }\end{array}$ \\
\hline 12 & $\begin{array}{l}\text { Con la experiencia } \\
\text { aprendizaje-servicio he } \\
\text { trabajado los aspectos que he } \\
\text { marcado como importantes } \\
\text { en la sección anterior }\end{array}$ & $1 b$ \\
\hline 13 & $\begin{array}{l}\text { Con la introducción teórica de } \\
\text { la sesión anterior ya supe lo } \\
\text { valioso que es el enfoque } \\
\text { aprendizaje-servicio }\end{array}$ & $1 b$ \\
\hline
\end{tabular}

La actividad práctica me ha servido para darle aún más valor al enfoqueaprendizajeservicio

El haber llevado a cabo una experiencia de aprendizajeservicio me ha servido para conocer la sensación que se tiene cuando se trabaja desde esta perspectiva

El haber llevado a cabo una experiencia de aprendizaje-

16 servicio ha sido positivo para mi desde un punto de vista de impacto social

El hecho de poner en práctica las fases de sentir e imaginar

17 me ha motivado más a llevarlo a la práctica en mis clases

Nota: Sección 2. Mi experiencia con aprendizajeservicio. Valora tu grado de acuerdo con las siguientes afirmaciones sobre tu experiencia en la sesión práctica de aprendizaje-servicio Fuente: elaboración propia

Tabla 3. Preguntas de la Sección 3 del cuestionario de investigación

\begin{tabular}{|c|c|c|}
\hline No & Preguntas & $\begin{array}{l}\text { Dimensión } \\
\text { de estudio }\end{array}$ \\
\hline 18 & $\begin{array}{l}\text { Antes del máster tenía una } \\
\text { idea clara sobre qué es } \\
\text { aprendizaje-servicio }\end{array}$ & $1 a$ \\
\hline 19 & $\begin{array}{l}\text { Actualmente tengo una idea } \\
\text { clara sobre qué es } \\
\text { aprendizaje-servicio }\end{array}$ & $1 a$ \\
\hline 20 & $\begin{array}{l}\text { Conozco las cuatro fases } \\
\text { principales del aprendizaje- } \\
\text { servicio y sé qué se hace en } \\
\text { cada una de ellas. }\end{array}$ & $1 a$ \\
\hline 21 & $\begin{array}{l}\text { Con los materiales y vídeos } \\
\text { de la web me siento } \\
\text { preparado para poner en } \\
\text { práctica una experiencia de } \\
\text { aprendizaje-servicio }\end{array}$ & $1 b$ \\
\hline 22 & $\begin{array}{l}\text { Con la formación recibida, } \\
\text { me siento a gusto para } \\
\text { poner en práctica una } \\
\text { experiencia de aprendizaje- } \\
\text { servicio }\end{array}$ & $1 b$ \\
\hline
\end{tabular}

Ortega-Ruipérez, B. (2020). Formación del profesorado a distancia sobre aprendizaje-servicio: aspectos a considerar. RIDAS, Revista Iberoamericana de Aprendizaje Servicio, 9, 102-122. 


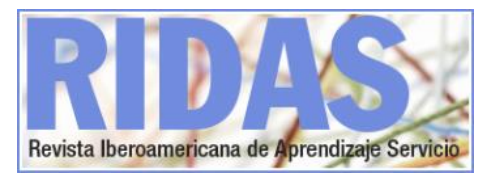

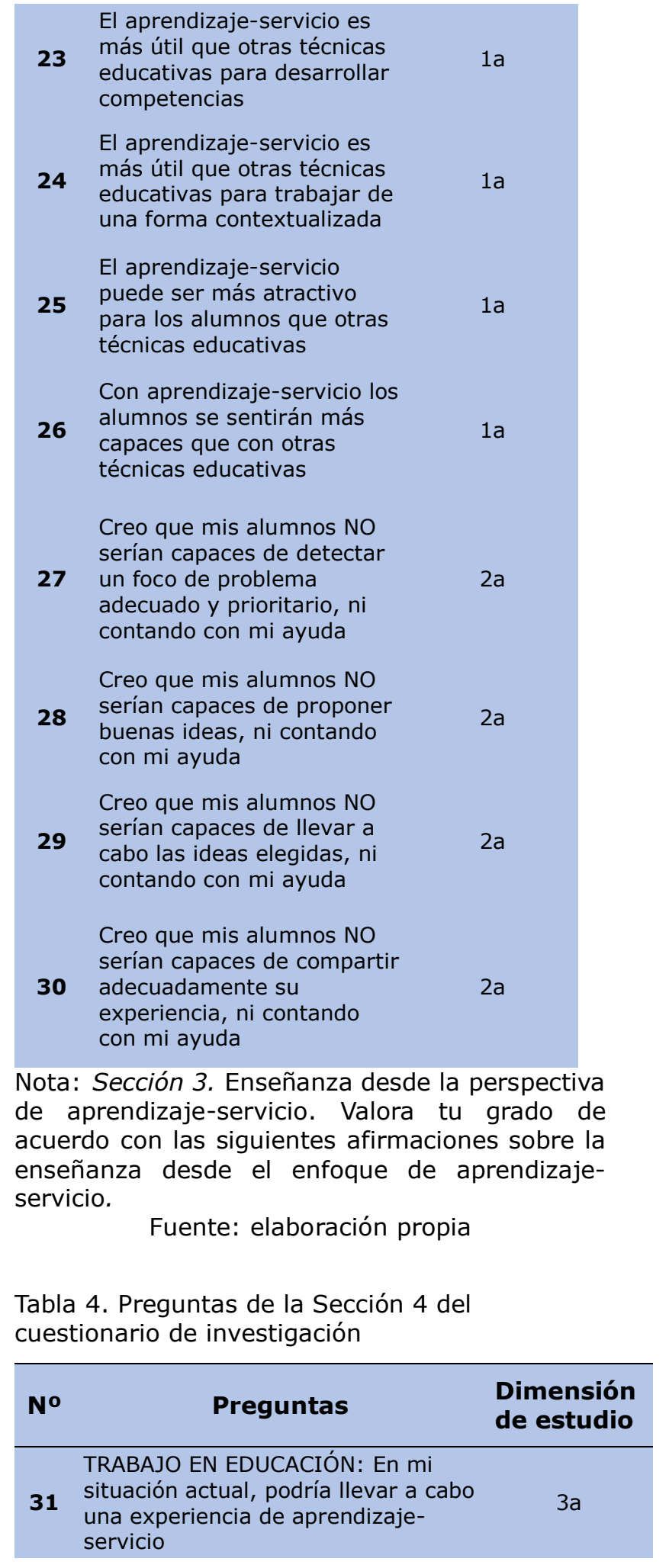

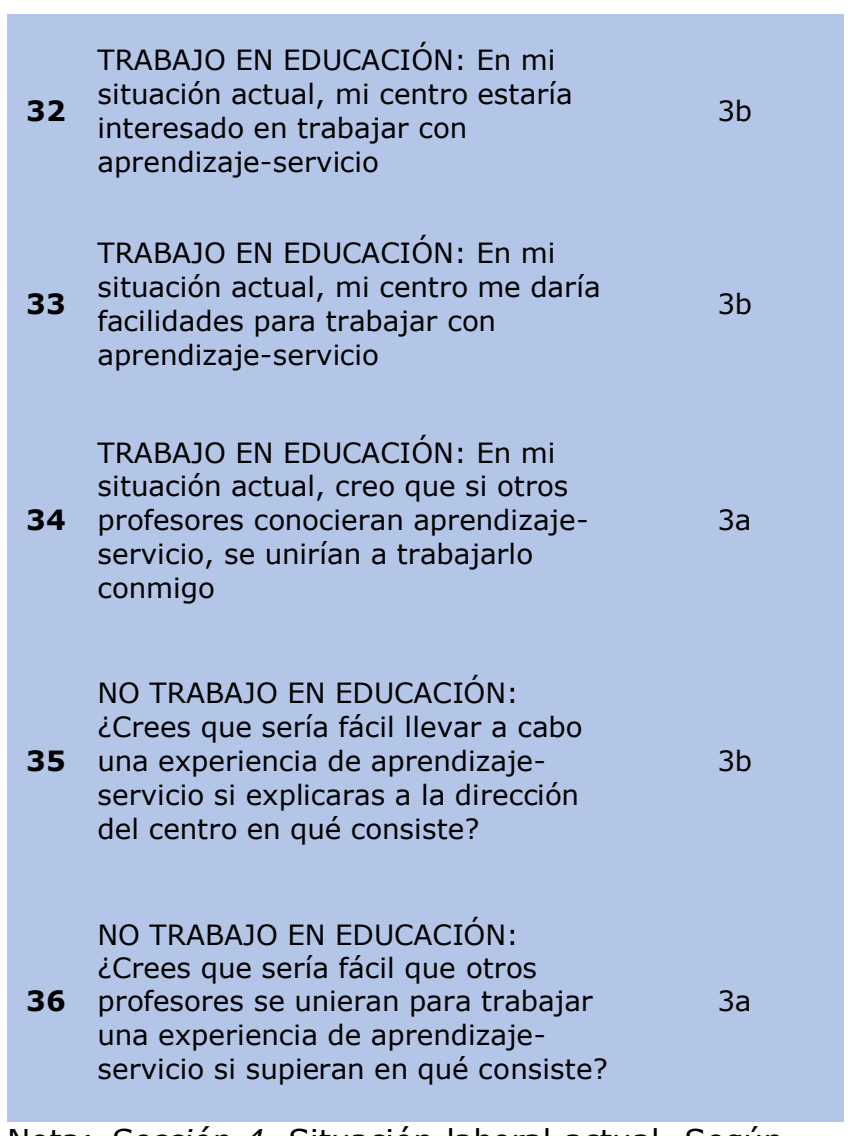

Nota: Sección 4. Situación laboral actual. Según tu respuesta a esta pregunta, el formulario te llevará a una sección que se ajuste a tu situación actual.

\section{Fuente: elaboración propia}

Tabla 5. Preguntas de la Sección 5 del cuestionario de investigación

\begin{tabular}{clc}
\hline No & \multicolumn{1}{c}{ Preguntas } & $\begin{array}{c}\text { Dimensión } \\
\text { de estudio }\end{array}$ \\
\hline 37 & $\begin{array}{l}\text { Valora tu satisfacción } \\
\text { respecto a la experiencia } \\
\text { llevada a cabo en clase }\end{array}$ & 1b \\
& $\begin{array}{l}\text { Valora tu satisfacción } \\
\text { respecto a esta forma de } \\
\text { enseñar con impacto social }\end{array}$ & $1 \mathrm{a}$ \\
\end{tabular}

Nota: Sección 5. Para terminar.

Fuente: elaboración propia

Ortega-Ruipérez, B. (2020). Formación del profesorado a distancia sobre aprendizaje-servicio: aspectos a considerar. RIDAS, Revista Iberoamericana de Aprendizaje Servicio, 9, 102-122. 
Tabla 6. Resumen de las preguntas incluidas por dimensión de estudio.

\begin{tabular}{|c|c|c|}
\hline No & Dimensión & $\begin{array}{l}\text { Número de } \\
\text { preguntas }\end{array}$ \\
\hline 1.a & $\begin{array}{l}\text { Valoración de este enfoque } \\
\text { educativo. }\end{array}$ & $\begin{array}{c}1,18,19,20,23 \\
24,25,26,38\end{array}$ \\
\hline 1.b & $\begin{array}{l}\text { Valoración de la } \\
\text { experiencia educativa } \\
\text { llevada en el aula. }\end{array}$ & $\begin{array}{c}12,13,14,15,16 \\
17,21,22,37\end{array}$ \\
\hline 2.a & $\begin{array}{l}\text { Posibilidades de desarrollar } \\
\text { ciertas habilidades en los } \\
\text { alumnos. }\end{array}$ & $\begin{array}{c}2,3,10,11,27 \\
28,29,30\end{array}$ \\
\hline 2.b & $\begin{array}{l}\text { Utilidad para mejorar la } \\
\text { concienciación social. }\end{array}$ & $4,5,6,7,8,9$ \\
\hline 3.a & $\begin{array}{l}\text { Comodidad de aplicación } \\
\text { de una técnica tan práctica } \\
\text { y dinámica. }\end{array}$ & $31,34,36$ \\
\hline 3.b & $\begin{array}{l}\text { Posibilidades reales de } \\
\text { llevar el aprendizaje- } \\
\text { servicio a la práctica en un } \\
\text { centro educativo. }\end{array}$ & $32,33,35$ \\
\hline
\end{tabular}

Fuente: elaboración propia

\section{Procedimiento}

En esta universidad de educación a distancia, la asistencia a las sesiones en directo es optativa, aunque se recompensa con una subida de $+0,1$ puntos en la calificación final por cada sesión a la que se asista en directo, con un máximo de 0,3 puntos.

Para esta experiencia práctica sobre aprendizaje-servicio llevada a cabo se emplearon dos sesiones presenciales completas, de una hora de duración cada una, ya que la cantidad de contenidos a impartir en cada

asignatura no permitía dedicar más tiempo a esta experiencia. Para todos aquellos alumnos que no pueden asistir en directo, la sesión queda grabada en el aula virtual.

En la primera sesión presencial se explicó de forma teórica en qué consisten las experiencias de aprendizaje-servicio y se compartió la web del proyecto Design for Change para que los alumnos pudieran entender mejor este enfoque educativo. En la web del proyecto aparecen 4 vídeos, uno por cada una de las fases propuestas en este proyecto. En esta sesión se visualizó el primero de los vídeos, correspondiente a la fase uno, Siente. En el vídeo se anima a explorar el ambiente y detectar focos de mejora o necesidades concretas. Se comunicó a los alumnos el objetivo de la siguiente sesión, la sesión práctica, llamando a la máxima asistencia posible a los alumnos. Se les pidió que pensaran necesidades y focos de mejora de su entorno escolar de cara a la siguiente sesión, la sesión en la que se llevaría a cabo la experiencia educativa.

Durante la sesión práctica, se llevó el esquema de Design for Change. A la primera fase se dedicaron los 15 primeros minutos de la sesión, los primeros 5 minutos se pusieron en común los focos detectados por los alumnos en sus respectivos centros educativos, en los siguientes 5 minutos se realizó una votación para seleccionar los focos de mejora que requerían una mayor urgencia de resolución, y los últimos 5 minutos de esta fase se utilizaron para hacer grupos que trabajasen los diferentes focos de 
mejora seleccionados. Los alumnos que asistían en ese momento a la sesión fueron repartidos aleatoriamente a los diferentes grupos en un número proporcional.

A la segunda fase, Imagina, se dedicaron los siguiente 20 minutos, en los primeros minutos se vio el vídeo correspondiente a esa fase, para que cada grupo empezara a proponer diferentes ideas para solucionar el foco a mejorar, en los minutos finales de esa fase (la docente dio un aviso cuando quedaban 5 minutos) debían elegir la o las mejores ideas (dependiendo del esfuerzo que lleve cada una de las ideas elegidas) para realizar.

La tercera fase, Haz, duró 5 minutos ya que no era posible llevarla a cabo. Por tanto, se visionó el vídeo de esta tercera fase y se dijo a los alumnos que imaginasen que habían llevado a cabo sus propuestas.

Por último, a la cuarta y última fase se dedicaron los 20 minutos restantes. En este tiempo se visualizó el vídeo de esta fase, y acto seguido empezaron a pensar qué imágenes o vídeos querrían compartir con el resto del mundo para mostrar el proyecto que habían Ilevado a cabo. Además, tenían que decidir entre los integrantes de cada grupo un mensaje para dar a conocer su aportación. Todas las ideas sobre cómo compartir el proyecto se compartieron en un documento común a toda la clase y se comentaron en los minutos finales de clase.

Cuando terminó la sesión práctica se explicó a los alumnos el estudio que se está llevando a cabo y se pidió amablemente la colaboración voluntaria de todos ellos, lo que ha dado lugar a los resultados obtenidos que se comentan a continuación.

\section{Resultados}

\subsection{Conocimiento general sobre aprendizaje-servicio}

Este primer análisis se ha realizado para obtener un mayor conocimiento de base que nos sirva de partida para entender las valoraciones que realizan los docentes sobre el aprendizajeservicio. Para ello, se han analizado los ítems de la tabla 6 .

Antes del máster, más de la mitad de los docentes apenas conocía el enfoque de aprendizaje-servicio. Según la pregunta 18 , el $26,2 \%$ no tenían conocimiento, respondiendo 1 sobre 5; y el $29,2 \%$ tenía un conocimiento básico sobre la técnica ( 2 sobre 5 ). Esto hace un total de $55,4 \%$ de los docentes, es decir, más de la mitad apenas tenía conocimiento sobre aprendizaje-servicio. Sin embargo, después de la experiencia llevada a cabo, el 43,1\% tiene una idea clara sobre qué es, marcando un 5 sobre 5 en la pregunta 19 , si a esto sumamos el $44,6 \%$ de los que marcaron un 4 sobre 5 , obtenemos que actualmente el $87,7 \%$ tiene una idea bastante clara sobre qué es el aprendizaje-servicio. Respecto al conocimiento sobre las 4 fases que se proponen en Design for Change, sumando las respuestas de $4 \mathrm{y}$ 5 sobre 5 , el $86,2 \%$ manifiesta saber qué acciones llevar a cabo en cada una de ellas.

En cuanto a la valoración de esta

Ortega-Ruipérez, B. (2020). Formación del profesorado a distancia sobre aprendizaje-servicio: aspectos a considerar. RIDAS, Revista Iberoamericana de Aprendizaje Servicio, 9, 102-122. 
técnica sobre su utilidad para desarrollar competencias, trabajar de forma contextualizada, motivar a los alumnos y mejorar la percepción de los alumnos sobre sus capacidades reales, los docentes han valorado más positivamente los beneficios de utilizar esta técnica por ser atractiva para los alumnos, con una media de 4,26 sobre 5 ; seguido de mejorar la percepción sobre las capacidades de los alumnos (4,08 sobre 5$)$ y de trabajar de forma contextualizada (4,06 sobre 5$)$. En último lugar, los docentes valoran la capacidad del aprendizaje-servicio para desarrollar competencias en los alumnos, con una media de 3,8 sobre 5 puntos.

Además, los docentes consideran fundamental aplicar el conocimiento al mundo real, con una puntuación media de 6,69 sobre 7 (el 76,9\% puntúo esta pregunta con la máxima puntuación). Un aspecto clave de esta técnica educativa.

En general, la valoración final que los docentes le dan al aprendizaje-servicio es de 8,78 sobre 10 , habiéndose distribuido las valoraciones como se muestra en la figura 2.

Por otra parte, se ha querido conocer la valoración de la experiencia educativa llevada en el aula, lo que permitirá mejorar la acción formativa para que los alumnos vean la importancia de utilizar técnicas educativas con impacto social. Las puntuaciones medias de todas las preguntas correspondientes a esta dimensión se pueden observar en la tabla 7.
Figura 2. Distribución de resultados con porcentajes de la pregunta 38

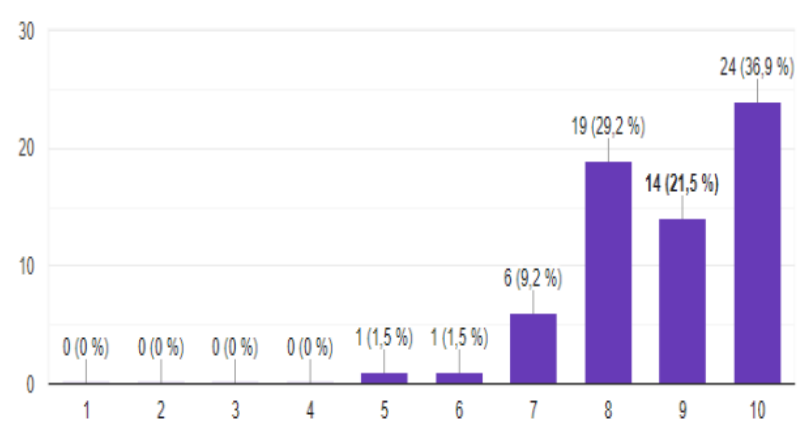

Fuente: elaboración propia

Tabla 7. Puntuaciones medias de las preguntas correspondientes a la dimensión $1 \mathrm{~b}$

\begin{tabular}{|c|c|c|}
\hline No & Preguntas & $\begin{array}{c}\text { Puntuación } \\
\text { media }\end{array}$ \\
\hline 12 & $\begin{array}{l}\text { Con la experiencia aprendizaje- } \\
\text { servicio he trabajado los aspectos } \\
\text { que he marcado como importantes } \\
\text { en la sección anterior }\end{array}$ & $4,138 / 5$ \\
\hline 13 & $\begin{array}{l}\text { Con la introducción teórica de la } \\
\text { sesión anterior ya supe lo valioso } \\
\text { que es el enfoque aprendizaje- } \\
\text { servicio }\end{array}$ & $4,246 / 5$ \\
\hline 14 & $\begin{array}{l}\text { La actividad práctica me ha servido } \\
\text { para darle aún más valor al } \\
\text { enfoque aprendizaje-servicio }\end{array}$ & $4,476 / 5$ \\
\hline 15 & $\begin{array}{l}\text { El haber llevado a cabo una } \\
\text { experiencia de aprendizaje-servicio } \\
\text { me ha servido para conocer la } \\
\text { sensación que se tiene cuando se } \\
\text { trabaja desde esta perspectiva }\end{array}$ & $4,261 / 5$ \\
\hline 16 & $\begin{array}{l}\text { El haber llevado a cabo una } \\
\text { experiencia de aprendizaje-servicio } \\
\text { ha sido positivo para mi desde un } \\
\text { punto de vista de impacto social }\end{array}$ & $4,369 / 5$ \\
\hline 17 & $\begin{array}{l}\text { El hecho de poner en práctica las } \\
\text { fases de sentir e imaginar me ha } \\
\text { motivado más a llevarlo a la } \\
\text { práctica en mis clases }\end{array}$ & $4,492 / 5$ \\
\hline
\end{tabular}




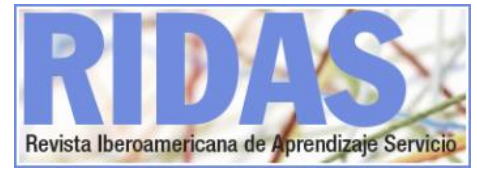

Con los materiales y vídeos de la

21 web me siento preparado para poner en práctica una experiencia de aprendizaje-servicio

Con la formación recibida, me siento a gusto para poner en práctica una experiencia de aprendizaje-servicio

Valora tu satisfacción respecto a la experiencia llevada a cabo en clase

$8,492 / 10$ que arroje esta investigación, mejorando la distribución de puntuaciones (figura 3).

Figura 3. Distribución de resultados con porcentajes de la pregunta 37

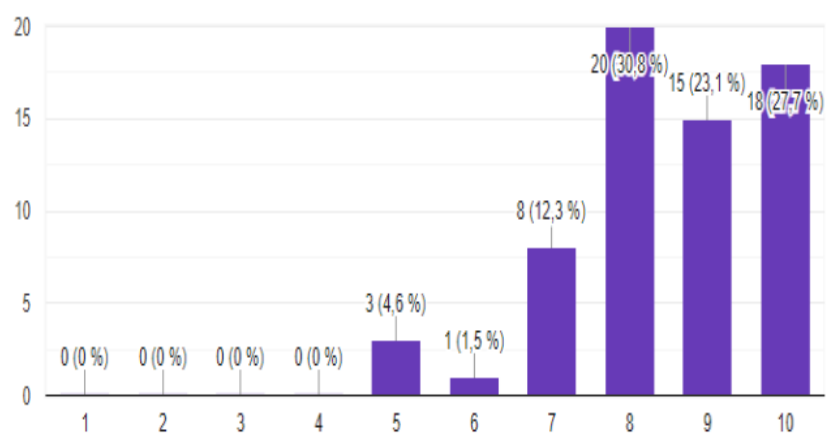

Fuente: elaboración propia

5.2 Valoración de los beneficios del aprendizaje-servicio

En segundo lugar, se ha querido conocer la valoración docente sobre algunos de los beneficios que más se estudian en la literatura relativa al aprendizaje-servicio.

Se ha estudiado, por una parte, las posibilidades de desarrollar ciertas habilidades en los alumnos. Las puntuaciones medias se encuentran en la tabla 8. Entre las habilidades que los docentes creen que más desarrollar trabajar desde un enfoque de aprendizaje-servicio son las habilidades de comunicación y las de trabajo, la primera con una media de 6,7 y la segunda de 6,63 sobre 7 . Les sigue el desarrollo de habilidades organizativas, $y$, por último, creen que las habilidades que menos se desarrollan son las relativas al liderazgo. 
El resto de las preguntas de esta dimensión están planteadas de forma negativa, esto quiere decir que la valoración positiva implica una menor puntuación. En este caso, todas las preguntas tienen una escala de respuesta sobre 5 puntos. Se ha planteado de esta forma para comprobar la confianza que tienen los docentes en sus alumnos en las 4 fases de DFC.

Los docentes creen que sus alumnos serían más capaces de proponer buenas ideas (fase 2. Imagina) y serían capaces de compartir los resultados en redes sociales (fase 4 . Comparte). Creen que serían ligeramente menos capaces para llevar a cabo las ideas elegidas (fase 3. Haz) y aún menos capaces para detectar los focos de problemas adecuados y prioritarios en su entorno (fase 1 . Siente).

Aún con estos resultados, las diferencias son mínimas $(0,3$ puntos de diferentes entre las medias de las preguntas mejor puntuadas y la pregunta peor puntuada), y todas ellas se sitúan por debajo de la puntuación media de la escala, es decir, por debajo de 2,5 sobre 5 .

Por otra parte, se ha estudiado la utilidad de esta técnica para mejorar la concienciación social en los alumnos (tabla 9). En este caso, los docentes valoran de forma más positiva la capacidad del aprendizaje-servicio para desarrollar habilidades para asumir la responsabilidad individual, con una media de 6,43 sobre 7; seguido del desarrollo de habilidades de responsabilidad social y ciudadana, con un 6,36 sobre 7 . El resto de preguntas se ha valorado desde los 0,3 puntos por debajo hasta los 0,6 puntos por debajo.

Tabla 8. Puntuaciones medias de las preguntas correspondientes a la dimensión $2^{a}$

\begin{tabular}{|c|c|c|}
\hline No & Preguntas & $\begin{array}{l}\text { Puntuación } \\
\text { media }\end{array}$ \\
\hline 2 & $\begin{array}{l}\text { Desarrollar habilidades de } \\
\text { trabajo }\end{array}$ & $6,630 / 7$ \\
\hline 3 & $\begin{array}{l}\text { Desarrollar habilidades de } \\
\text { organización }\end{array}$ & $6,492 / 7$ \\
\hline 10 & $\begin{array}{l}\text { Desarrollar habilidades de } \\
\text { liderazgo }\end{array}$ & $6,015 / 7$ \\
\hline 11 & $\begin{array}{l}\text { Desarrollar habilidades de } \\
\text { comunicación }\end{array}$ & $6,707 / 7$ \\
\hline 27 & $\begin{array}{l}\text { Creo que mis alumnos NO serían } \\
\text { capaces de detectar un foco de } \\
\text { problema adecuado y prioritario, } \\
\text { ni contando con mi ayuda }\end{array}$ & $2,369 / 5$ \\
\hline 28 & $\begin{array}{l}\text { Creo que mis alumnos NO serían } \\
\text { capaces de proponer buenas } \\
\text { ideas, ni contando con mi ayuda }\end{array}$ & $2,061 / 5$ \\
\hline 29 & $\begin{array}{l}\text { Creo que mis alumnos NO serían } \\
\text { capaces de llevar a cabo las } \\
\text { ideas elegidas, ni contando con } \\
\text { mi ayuda }\end{array}$ & $2,107 / 5$ \\
\hline 30 & $\begin{array}{l}\text { Creo que mis alumnos NO serían } \\
\text { capaces de compartir } \\
\text { adecuadamente su experiencia, } \\
\text { ni contando con mi ayuda }\end{array}$ & $2,061 / 5$ \\
\hline
\end{tabular}

Fuente: elaboración propia

Tabla 9. Puntuaciones medias de las preguntas correspondientes a la dimensión $2 \mathrm{~b}$

\begin{tabular}{clc}
\hline No & Preguntas & $\begin{array}{c}\text { Puntuación } \\
\text { media }\end{array}$ \\
\hline $\mathbf{4}$ & $\begin{array}{l}\text { Entender las diferencias } \\
\text { culturales y raciales }\end{array}$ & $6,061 / 7$ \\
$\mathbf{5}$ & $\begin{array}{l}\text { Desarrollar habilidades de } \\
\text { responsabilidad social y } \\
\text { ciudadana }\end{array}$ & $6,369 / 7$ \\
\hline
\end{tabular}


Desarrollar habilidades para

6 marcar una diferencia en la comunidad

7 Desarrollar una confianza social $6,076 / 7$ Desarrollar habilidades para

8 asumir la responsabilidad individual

Desarrollar habilidades para

9 ganarse la confianza de los demás

Fuente: elaboración propia

5.3 Valoración de los inconvenientes del aprendizaje-servicio

Por último, se ha realizado una valoración de los docentes sobre los inconvenientes más repetidos en la literatura sobre aprendizaje-servicio. Las preguntas de esta dimensión han sido diferentes para los docentes que se encuentran trabajando en educación y para los docentes que actualmente no trabajan en educación. De los 65 participantes, 61 de ellos están trabajando y sólo 4 de ellos no trabajan en educación.

Por una parte, en el caso de los docentes que están trabajando actualmente, se ha preguntado por la comodidad de aplicación de una técnica tan práctica y dinámica, por si podrían llevar a cabo una experiencia de este tipo y si creen que otros profesores se unirían a trabajar este tipo de experiencias si conocieran el aprendizaje-servicio. En estas dos preguntas, las puntuaciones medias han sido 3,85 y 3,73 sobre 5 , respectivamente. También, si creen que el centro estaría interesado en trabajarlo y si, en ese caso, les daría facilidades. En estos casos, las puntuaciones medias han descendido hasta 3,6 y 3,31 respectivamente. Aquí vemos cómo los docentes estarían más dispuestos, pero serían los centros quienes no estarían tan dispuestos y menos aún si tienen que dar facilidades.

En el caso de los docentes que no están trabajando actualmente, se pregunta si cree que sería fácil introducir aprendizaje-servicio en un centro si se explica a la dirección en qué consiste y si creen que otros profesores se animarían a realizar esta técnica si supieran qué es. En este caso, las respuestas han sido mucho más positivas, obteniendo puntuaciones medias de 4,25 y 4,5 sobre 5 , respectivamente.

\subsection{Discusión de resultados}

Respecto a la primera dimensión de estudio, en general, la valoración sobre esta técnica educativa es positiva. Lo que más valoran los docentes es el atractivo que supone para los alumnos esta técnica, también valoran en menor medida que los alumnos se sienten más capaces de hacer cosas y que trabajan de forma contextualizada. Sin embargo, no se valora como se esperaba el desarrollo de competencias en los alumnos. Esto nos hace reflexionar sobre la formación, para incorporar información sobre cómo podemos desarrollar determinadas competencias en nuestros alumnos con esta técnica.

Por otra parte, lo más relevante para esta investigación es poder aplicar los conocimientos aquí obtenidos en la 
mejora de la experiencia formativa. En general, la formación ha sido bien valorada, aunque se esperan obtener mejores resultados en formaciones futuras. Los docentes consideran que la parte práctica ha sido la más valiosa y valoran por encima de todo el haber puesto en práctica las fases de sentir e imaginar. Lo peor valorado ha sido el material subido a la plataforma, lo que será relativamente fácil de solucionar de cara a la siguiente formación a través de la búsqueda y confección de nuevos materiales. Otro resultado que llama la atención es que, aún con la formación recibida no se sienten del todo capaces de aplicarla en sus clases, esto puede mejorar proponiendo una actividad en la evaluación continua en la que tengan que plantear un proyecto fase a fase.

En cuanto a la segunda dimensión de estudio, la valoración que hacen los docentes sobre los beneficios del aprendizaje-servicio es, en general, positiva en el desarrollo de habilidades. Respecto a los dos grandes grupos de habilidades estudiados: habilidades de trabajo, comunicación, organización y liderazgo, por una parte, y habilidades relacionadas con la concienciación social en los alumnos por otra parte; los docentes valoran el aprendizaje-servicio como una técnica más adecuada para desarrollar habilidades de comunicación y trabajo, seguidas por las habilidades sociales relacionadas con la responsabilidad individual, social y ciudadana. Si obtenemos la media de valoración de las competencias de trabajo y relacionadas frente a la valoración de las competencias con carácter más social, las competencias de trabajo obtienen una media mayor $(6,45$ frente a 6,2 sobre 7$)$. La media de las competencias de trabajo y relacionadas supera incluso a las mayores puntuaciones de las preguntas de competencia social: responsabilidad individual y responsabilidad social y ciudadana.

Esto hace reflexionar de nuevo sobre cómo enfocar la formación. Se debe resaltar más el carácter social de esta técnica, a pesar de que la fase de siente nos ayuda a pensar en necesidades de nuestro entorno, quizá las soluciones que se proponen en la fase 2, Imagina, no son las que más impacto social pueden conseguir. Hay que estudiar más a fondo sobre esta cuestión, sobre la orientación que debemos ofrecer a los alumnos en la segunda fase, imagina.

Por último, la tercera dimensión de estudio, la valoración que hacen los docentes sobre los inconvenientes del aprendizaje-servicio, principalmente sobre la comodidad para llevarlo a la práctica y las posibilidades reales de aplicarlo. Se aprecia en general que los docentes que no están trabajando actualmente en ningún centro educativo son más optimistas que sus colegas que sí están trabajando actualmente, respecto a ambas cuestiones. Bastantes docentes que están en activo actualmente creen que podrían llevar a cabo una experiencia de aprendizajeservicio, y algunos de ellos contando incluso con otros profesores. Sin embargo, al preguntar sobre si el centro facilitaría la realización de la experiencia, el grado de acuerdo baja considerablemente. En este sentido, quizá la creación de material de 
información para la dirección de los centros podría ser un buen comienzo para poder realizar más experiencias de este tipo.

\section{Conclusiones}

Como conclusión se hace evidente la necesidad de aplicar de forma práctica una experiencia de formación de aprendizaje-servicio para mejorar la difusión online de esta técnica, aunque sea de forma simulada. Los resultados han coincido en líneas generales con los obtenidos por Caire (2019) sobre las altas valoraciones de la experiencia.

Sin embargo, se requiere la elaboración de materiales formativos que faciliten la aplicación del aprendizaje-servicio, ya que lo que encontramos actualmente son presentaciones con información básica o artículos de experiencias concretas. Entre ellos se deben encontrar materiales para informar a la dirección de los centros, de forma que faciliten la realización de estas experiencias, incluyéndolas en sus proyectos educativos de centro. Además, se debe profundizar más en cómo podemos desarrollar determinadas habilidades en los alumnos a través de esta técnica, de acuerdo con Strait, Turk y Nordyke (2015).

Por último, se debe considerar incluir en la evaluación continua la realización de una actividad práctica en la que simulen las diferentes fases, para asegurar que los alumnos de formación en línea aplican el aprendizaje-servicio y les permite comprenderlo mejor. Esta iniciativa podría mejorar la autoreflexión que echan en falta
Lehman y Conceição (2010) en su estudio sobre experiencias en línea.

Una de las principales limitaciones de este estudio ha sido la baja participación en la sesión presencial en la que se llevó a cabo la experiencia educativa. Al ser una universidad cuyas sesiones se graban para que los alumnos puedan visualizarlas en el momento que deseen, la asistencia a las sesiones es relativamente baja, no llegando al $50 \%$ en muchas ocasiones.

Una futura línea de investigación, además de la investigación principal que se lleva a cabo sobre formación online, será realizar la misma experiencia práctica de aprendizajeservicio en la educación superior presencial, con el fin de comprobar si existen diferencias en cuanto a las percepciones y la valoración de los docentes en formación sobre la utilidad $y$, en general, los beneficios e inconvenientes de este enfoque educativo con impacto social. Para ello se impartirá una formación similar, con la simulación de la fase 3, Haz, en la que imaginarán las acciones que llevarían a cabo, debido al ajustado tiempo de clase del que se dispone en una asignatura de máster que no se destina exclusivamente a tratar el aprendizaje-servicio.

\section{Referencias bibliográficas}

Caire, M. (2019). Contribuciones de las experiencias de aprendizaje-servicio desde la perspectiva de estudiantes de la Universidad Alberto Hurtado. RIDAS, Revista Iberoamericana de Aprendizaje Servicio, 7 89-111.

Ortega-Ruipérez, B. (2020). Formación del profesorado a distancia sobre aprendizaje-servicio: aspectos a considerar. RIDAS, Revista Iberoamericana de Aprendizaje Servicio, 9, 102-122. 
De Castro, A., Martínez, A., y Guerra, D. (2017). Aprendizaje Servicio, investigación de aula y desarrollo docente. En A. De Castro, y Domínguez Merlano, E. (Comps.) Transformar para educar 4. Aprendizaje Servicio (pp. 4166). Colombia: Universidad del Norte. Recuperado de https://dialnet.unirioja.es/servlet/libro? codigo $=705350$

DFC World. (sf.). Design for Change, Our Story: Timeline. Boston, United States of America .Recuperado de https://dfcworld.com/SITE/dfcstory

Duque, E. (2018). Evaluando una experiencia de aprendizaje servicio en torno al aprendizaje de conceptos de la ciudadanía digital. RIDAS, Revista Iberoamericana de Aprendizaje Servicio, 5, 12-23.

Esparza, M., Morín, V. y Rubio, L. (2018). La incorporación del aprendizaje-servicio en la universidad: la experiencia de la Universidad de Barcelona. RIDAS, Revista Iberoamericana de Aprendizaje Servicio, 6, 97-114.

Fisch, K., McLeod, S., y Bronman, J. (2013). Why Design Thinking in Education. Consider the Current Scenario. En DFC Toolbox of Change (Ed.) Design for Change. Teacher's Manual: Teaching \& Assessing the 21st century skills. India: Riverside.

Fuertes, M. (2013). El ApS en el prácticum de la formación inicial del profesorado. Propuesta de una estrategia de docencia y aprendizaje para la adquisición de competencias genéricas claves para la formación y el desempeño profesional (Tesis doctoral). Universitat Internacional de Catalunya, Barcelona, España.

Hébert, A., y Hauf, P. (2015). Student learning through service learning: Effects on academic development, civic responsibility, interpersonal skills and practical skills. Active Learning in Higher Education, 16 (1), 37-49. Recuperado de https://journals.sagepub.com/doi/abs/1 $0.1177 / 1469787415573357$

Lehman, R., \& Conceição, S. (2010). Creating a sense of presence in online teaching: How to "be there" for distance learners. San Francisco, United States of America: John Wiley \& Sons.

López, I. y Benítez, J. (2018). El Aprendizaje Servicio en la Universidad: una experiencia en el marco de una asignatura del Grado en Educación Primaria. REDU, Revista de docencia Universitaria, 16(2), 195-210.

Recuperado de https://dialnet.unirioja.es/servlet/articu lo? codigo $=6726715$

Malvey, D., Hamby, E., \& Fottler, M. (2006). E-service learning: A pedagogic innovation for healthcare management education. Journal of Health Administration Education, 33(2), 181198. Recuperado de https://europepmc.org/abstract/med/1 6700443

Martín, E., Solari, M., De Vicente, J., Luque, M.J., Nieto, M., y Coll, C. (2018). La potencialidad del aprendizaje servicio para la personalización del aprendizaje escolar. RIDAS, Revista Iberoamericana de 
Aprendizaje Servicio, (5), 37-61.

Martínez, A. (2018). El aprendizaje servicio como experiencia en el Grado de Educación Primaria. RIDAS, Revista Iberoamericana de Aprendizaje Servicio, (5), 142-149.

McWhorter, R., Delello, J., y Roberts, P. (2016). Giving back: exploring serviceLearning in an online learning environment. Journal of Interactive Online Learning, 14 (2), 80-99.

Recuperado de http://hdl.handle.net/10950/494

Miyazaki, T., Anderson, J. B., \& Jones, S. (2017). The Influence of ServiceLearning on the Civic Attitudes and Skills of Japanese Teacher Education Candidates. International Journal of Research on Service-Learning in Teacher Education, 5(1), 1-10. Recuperado de https://ijrslte.journal.library.uta.edu/in dex.php/IJRSLTE/article/view/54

Páez, M. y Puig, J., (2013) La reflexión en el Aprendizaje-Servicio. Revista internacional de educación para la justifica social, 2(2), 13-32. Recuperado de

https://repositorio.uam.es/handle/1048 6/660355

Pérez, L. y Ochoa, A. (2017). El aprendizaje-Servicio (APS) como estrategia para educar en la ciudadanía. Alteridad. Revista de educación, 12 (2), 175-187.

Puig, J., Gijón, M., Martín, X. y Rubio, L. (2011). Aprendizaje-servicio y educación para la ciudadanía. Revista de Educación, número extraordinario,
45-67. Recuperado de

http://www.revistaeducacion.educacion .es/re2011/re2011_03.pdf

Rodríguez, M. (2014). El AprendizajeServicio como estrategia metodológica en la Universidad. Revista Complutense de Educación, 25(1), 95-113.

Recuperado de

https://idus.us.es/xmlui/handle/11441/ 43001

Sotelino, A., Santos, M. y García, J. (2019). El aprendizaje-servicio como vía para el desarrollo de competencias interculturales en la Universidad. Educatio Siglo XXI, 37(1), 73-90.

Strait, J., Turk, J. y Nordyke, K. (2015). Pedagogy of civic engagement, highimpact practices, and eServiceLearning. En J. Strait y K. Nordyke (Eds.) eService-Learning. Creating experiential learning and civic engagement through online and hybrid courses (pp. 7-19). Virginia, United States of America: Stylus Publishing, LLC.

Toncar, M., Reid, J., Burns, D., Anderson, C. y Nguyen, H. (2006). Uniform assessment of the benefits of service learning: The development, evaluation, and implementation of the SELEB scale. Journal of Marketing Theory and Practice, 14(3), 223-238. Recuperado de https://www.tandfonline.com/doi/abs/1 0.2753/MTP1069-6679140304

Waldner, L., McGorry, S. y Widener, M. (2010). Extreme E-Service Learning (XE-SL): E-Service Learning in the $100 \%$ Online Course. MERLOT Journal of Online Learning and Teaching, 6(4), 
839-851. Recuperado de

http://jolt.merlot.org/vol6no4/waldner

1210.pdf

Waldner, L., McGorry, S. \& Widener, M.

(2012). E-Service Learning: the

evolution of Service-Learning to engage

a growing online student population.

Journal of Higher Education Outreach

and Engagement, 16(2), 123-150.

Recuperado de

http://openjournals.libs.uga.edu/index.

php/jheoe/article/view/792 